\title{
INEQUALITIES APPLICABLE TO RETARDED VOLTERRA INTEGRAL EQUATIONS
}

\author{
B. G. PACHPATTE
}

\begin{abstract}
The main objective of this paper is to establish explicit bounds on certain integral inequialities which can be used as tools in the study of certain classes of retarded Volterra integral equations.
\end{abstract}

\section{Introduction}

In 1972, K. Ahmedov, A. Jakubov and A. Veisov obtained the upper bounds on the following inequalities. (see $[2$, pp.11, 13])

$$
\begin{aligned}
& u(t) \leq f(t)+\int_{t_{0}}^{\phi(t)} k(t, s) g(u(s)) d s, \\
& u(t) \leq f(t)+\sum_{i=1}^{n} a_{i}(t) \int_{t_{0}}^{\phi_{i}(t)} b_{i}(s) g(u(s) d s),
\end{aligned}
$$

under some suitable conditions on the functions involved in (1.1) and (1.2). The bounds obtained on (1.1) and (1.2) can be used to study the qualitative behavior of solutions of certain retarded integral equations. In fact, the bound obtained on (1.2) is based on the solutions of a certain initial value problem (see [2, Theorem 24, p.13]), which basically involves the comparision principle and usually difficult to calculate explicitly. In the present paper, we offer some fundamental integral inequalities of the above forms providing explicit bounds, which can be used as tools in the study of certain classes of Volterra integral equations involving several retarded arguments. To convey the importance of the results, applications of one of our results are also given.

\section{Statement of Results}

In what follows $R$ denotes the set of real numbers, $R_{+}=[0, \infty), I=\left[t_{0}, T\right)$ are the given subsets of $R$ and define $H=\left\{(t, s): t_{0} \leq s \leq t<T\right\}$. Throughout, all the integrals involved are assumed to exist on the respective domains of their definitions.

Received April 15, 2003; revised June 11, 2003.

2000 Mathematics Subject Classification. 26D15, 26D20.

Key words and phrases. Explicit bounds, integral inequalities, Volterra integral equations, quatitative behavior, comparision principle, retarded arguments. 
Our main results are given in the following theorems.

Theorem 2.1. Let $u(t), a(t) \in C\left(I, R_{+}\right) k_{i}(t, s), \frac{\partial}{\partial t} k_{i}(t, s) \in C\left(H, R_{+}\right)$and $\alpha_{i}(t) \in$ $C^{1}(I, I)$ be nondecreasing with $\alpha_{i}(t) \leq t$ on $I$ for $i=1,2, \ldots, n$.

$\left(\mathrm{a}_{1}\right)$ If $c \geq 0$ is a constant and

$$
u(t) \leq c+\sum_{i=1}^{n} \int_{\alpha_{i}\left(t_{0}\right)}^{\alpha_{i}(t)} k_{i}(t, s) u(s) d s,
$$

$t \in I$, then

$$
u(t) \leq c \exp \left(\int_{t_{0}}^{t} Q(s) d s\right)
$$

for $t \in I$, where

$$
Q(t)=\sum_{i=1}^{n}\left[k_{i}\left(t, \alpha_{i}(t)\right) \alpha_{i}^{\prime}(t)+\int_{\alpha_{i}\left(t_{0}\right)}^{\alpha_{i}(t)} \frac{\partial}{\partial t} k_{i}(t, \sigma) d \sigma\right]
$$

for $t \in I$.

$\left(\mathrm{a}_{2}\right)$ If $a(t)$ is nondecreasing for $t \in I$ and

$$
u(t) \leq a(t)+\sum_{i=1}^{n} \int_{\alpha_{i}\left(t_{0}\right)}^{\alpha_{i}(t)} k_{i}(t, s) u(s) d s
$$

for $t \in I$ then

$$
u(t) \leq a(t) \exp \left(\int_{t_{0}}^{t} Q(s) d s\right)
$$

for $t \in I$ where $Q(t)$ is given by (2.3).

Theorem 2.2. Let $u(t), a(t), k_{i}(t, s), \frac{\partial}{\partial t} k_{i}(t, s), \alpha_{i}(t)$ be as in Theorem 2.1 .

$\left(\mathrm{b}_{1}\right)$ Let $c \geq 0$ be a constant, $g(u)$ be continous, nondecreasing function defined on $R_{+}$ and $g(u)>0$ on $(0, \infty)$. If

$$
u(t) \leq c+\sum_{i=1}^{n} \int_{\alpha_{i}\left(t_{0}\right)}^{\alpha_{i}(t)} k_{i}(t, s) g(u(s)) d s,
$$

for $t \in I$, then for $t_{0} \leq t \leq t_{1}, t, t_{1} \in I$

$$
u(t) \leq G^{-1}\left[G(c)+\int_{t_{0}}^{t} Q(s) d s\right]
$$

where $Q(t)$ is given by $(2.3)$ and $G^{-1}$ is the inverse function of

$$
G(r)=\int_{r_{0}}^{r} \frac{d s}{g(s)}, \quad r>0,
$$


$r_{0}>0$ is arbitrary and $t_{1} \in I$ is chosen so that

$$
G(c)+\int_{t_{0}}^{t} Q(s) d s \in \operatorname{Dom}\left(G^{-1}\right)
$$

for all $t$ laying in the interval $t_{0} \leq t \leq t_{1}$

$\left(\mathrm{b}_{2}\right)$ Let $g(u)$ be as in $\left(\mathrm{b}_{1}\right)$ and suppose in addition it is subadditive. If

$$
u(t) \leq a(t)+\sum_{i=1}^{n} \int_{\alpha_{i}\left(t_{0}\right)}^{\alpha_{i}(t)} k_{i}(t, s) g(u(s)) d s,
$$

for $t \in I$ then for $t_{0} \leq t \leq t_{2}, t, t_{2} \in I$

$$
u(t) \leq a(t)+G^{-1}\left[G(B(t))+\int_{t_{0}}^{t} Q(s) d s\right],
$$

where $G, G^{-1}, Q$ be as in $\left(\mathrm{b}_{1}\right)$,

$$
B(t)=\sum_{i=1}^{n} \int_{\alpha_{i}\left(t_{0}\right)}^{\alpha_{i}(t)} k_{i}(t, s) g(a(s)) d s
$$

for $t \in I$ and $t_{2} \in I$ is chosen so that

$$
G(B(t))+\int_{t_{0}}^{t} Q(s) d s \in \operatorname{Dom}\left(G^{-1}\right),
$$

for all $t \in I$ laying in the interval $t_{0} \leq t \leq t_{2}$.

\section{Proofs of Theorem 2.1 and 2.2}

From the hypotheses, we observe that $\alpha_{i}^{\prime} \geq 0$ for $t \in I$. and

$\left(\mathrm{a}_{1}\right)$ Define a function $z(t)$ by the right hand side of $(2.1)$. Then $z\left(t_{0}\right)=c, u(t) \leq z(t)$

$$
\begin{aligned}
z^{\prime}(t) & =\sum_{i=1}^{n}\left\{k_{i}\left(t, \alpha_{i}(t)\right) u\left(\alpha_{i}(t)\right) \alpha_{i}^{\prime}(t)+\int_{\alpha_{i}\left(t_{0}\right)}^{\alpha_{i}(t)} \frac{\partial}{\partial t} k_{i}(t, s) u(s) d s\right\} \\
& \leq\left\{\sum_{i=1}^{n} k_{i}\left(t, \alpha_{i}(t)\right) z\left(\alpha_{i}(t)\right) \alpha_{i}^{\prime}(t)+\int_{\alpha_{i}\left(t_{0}\right)}^{\alpha_{i}(t)} \frac{\partial}{\partial t} k_{i}(t, s) z(s) d s\right\} \\
& \leq\left\{\sum_{i=1}^{n} k_{i}\left(t, \alpha_{i}(t)\right) \alpha_{i}^{\prime}(t)+\int_{\alpha_{i}\left(t_{0}\right)}^{\alpha_{i}(t)} \frac{\partial}{\partial t} k_{i}(t, s) d s\right\} z\left(\alpha_{i}(t)\right) \\
& \leq Q(t) z(t),
\end{aligned}
$$

which implies

$$
z(t) \leq c \exp \left(\int_{t_{0}}^{t} Q(s) d s\right)
$$


Using (3.1) in $u(t) \leq z(t)$ we get the desired inequality in (2.2).

$\left(\mathrm{a}_{2}\right)$ First we assume that $a(t)>0$ for all $t \in I$. It is easy to observe that for $s \leq \alpha_{i}(t) \leq t$ we have $a(s) \leq a\left(\alpha_{i}(t)\right) \leq a(t)$. In view of this, from (2.4) we observe that

$$
\frac{u(t)}{a(t)} \leq 1+\sum_{i=1}^{n} \int_{\alpha_{i}\left(t_{0}\right)}^{\alpha_{i}(t)} k_{i}(t, s) \frac{u(s)}{a(s)} d s .
$$

Now an application of the inequality in part $\left(\mathrm{a}_{1}\right)$ to (3.2) yields (2.5). If $a(t)=0$, then from (2.4) we observe that

$$
u(t) \leq \varepsilon+\sum_{i=1}^{n} \int_{\alpha_{i}\left(t_{0}\right)}^{\alpha_{i}(t)} k_{i}(t, s) u(s) d s,
$$

where $\varepsilon$ is an arbitrary small constant. An application of the inequality in part $\left(\mathrm{a}_{1}\right)$ yields

$$
u(t) \leq \varepsilon \exp \left(\int_{t_{0}}^{t} Q(s) d s\right) .
$$

Now by letting $\varepsilon \rightarrow 0$ in (3.3) we have $u(t)=0$ and hence (2.5) holds.

$\left(\mathrm{b}_{1}\right)$ We first assume that $c>0$ and define a function $z(t)$ by the right hand side of (2.6). Then $z\left(t_{0}\right)=c, u(t) \leq z(t)$ and

$$
\begin{aligned}
z^{\prime}(t) & =\sum_{i=1}^{n}\left\{k_{i}\left(t, \alpha_{i}(t)\right) g\left(u\left(\alpha_{i}(t)\right)\right) \alpha_{i}^{\prime}(t)+\int_{\alpha_{i}\left(t_{0}\right)}^{\alpha_{i}(t)} \frac{\partial}{\partial t} k_{i}(t, s) g(u(s)) d s\right\} \\
& \leq\left\{\sum_{i=1}^{n} k_{i}\left(t, \alpha_{i}(t)\right) g\left(z\left(\alpha_{i}(t)\right)\right) \alpha_{i}^{\prime}(t)+\int_{\alpha_{i}\left(t_{0}\right)}^{\alpha_{i}(t)} \frac{\partial}{\partial t} k_{i}(t, s) g(z(s)) d s\right\} \\
& \leq Q(t) g\left(z\left(\alpha_{i}(t)\right),\right. \\
& \leq Q(t) g(z(t)) .
\end{aligned}
$$

From (2.8) and (3.4) we have

$$
\begin{aligned}
\frac{d}{d t} G(z(t)) & =\frac{z^{\prime}(t)}{g(z(t))} \\
& \leq Q(t) .
\end{aligned}
$$

Now by setting $t=s$ in (3.5) and integrating it from $t_{0}$ to $t$ we have

$$
\begin{aligned}
& G(z(t))-G(c) \leq \int_{t_{0}}^{t} Q(s) d s \\
& z(t) \leq G^{-1}\left[G(c)+\int_{t_{0}}^{t} Q(s) d s\right]
\end{aligned}
$$

Using (3.7) in $u(t) \leq z(t)$ gives the required inequality in (2.7). 
If $c$ is nonnegative, we carry out the above procedure with $c+\varepsilon$ instead of $c$, where $\varepsilon>0$ is an arbitrary small constant and subsequently pass to the limit $\varepsilon \rightarrow 0$ to obtain (2.7). The subinterval $t_{0} \leq t \leq t_{1}$ is obvious.

$\left(\mathrm{b}_{2}\right)$ Define a function $z(t)$ by

$$
z(t)=\sum_{i=1}^{n} \int_{\alpha_{i}\left(t_{0}\right)}^{\alpha_{i}(t)} k_{i}(t, s) g(u(s)) d s .
$$

Then $z\left(t_{0}\right)=0$ and from $(2.9)$ we have

$$
u(t) \leq a(t)+z(t)
$$

Using (3.9) in (3.8) we have

$$
\begin{aligned}
z(t) & \leq \sum_{i=1}^{n} \int_{\alpha_{i}\left(t_{0}\right)}^{\alpha_{i}(t)} k_{i}(t, s) g(a(s)+z(s)) d s, \\
& \leq B(t)+\sum_{i=1}^{n} \int_{\alpha_{i}\left(t_{0}\right)}^{\alpha_{i}(t)} k_{i}(t, s) g(z(s)) d s
\end{aligned}
$$

where $B(t)$ is given by $(2.11)$. It is easy to observe that $B(t)$ is nonnegative and nondecreasing in $t$. Now by following the similar arguments as in the proof of Theorem 2.4.2 given in [4] with suitable modifications we get

$$
z(t) \leq G^{-1}\left[G(B(t))+\int_{t_{0}}^{t} Q(s) d s\right]
$$

Using (3.10) in (3.9) we get the required inequality in (2.10).

\section{Applications}

In this setction we present some model applications which display the importance of our results to the literature. Consider the following Volterra integral equation involving several retarded arguments

$$
x(t)=f(t)+\int_{t_{0}}^{t} F\left(t, s, x\left(s-h_{1}(s)\right), \ldots, x\left(s-h_{n}(s)\right)\right) d s
$$

where $f \in C(I, R), F \in C\left(I \times R^{n}, R\right)$ and $h_{i} \in C^{1}\left(I, R_{+} 1\right)$ be nonincreasing, $t-h_{i}(t) \in$ $C^{1}(I, I), h_{i}\left(t_{0}\right)=0, t-h_{i}(t) \geq 0, h_{i}^{\prime}(t)<1$ and

$$
M_{i}=\max _{t \in I} \frac{1}{1-h_{i}^{\prime}(t)} \quad \text { for } i=1,2, \ldots, n .
$$

The following theorem deals with estimate on the solution of equation (4.1). 
Theorem 4.1. Suppose that

$$
\begin{aligned}
|f(t)| & \leq c \\
\left|F\left(t, s, u_{1}, \ldots, u_{n}\right)\right| & \leq \sum_{i=1}^{n} k_{i}(t, s)\left|u_{i}\right|
\end{aligned}
$$

where $c$ and $k_{i}(t, s)$ are as in Theorem 1 . If $x(t)$ is any solution of $(4.1)$, then

$$
|x(t)| \leq c \exp \left(\int_{t_{0}}^{t} \bar{Q}(s) d s\right)
$$

for $t \in I$ where $\bar{Q}(t)$ is defined by the right hand side of $(2.3)$, replacing $k_{i}(t, r)$ and $\alpha_{i}(t)$ by $k_{i}\left(t, r+h_{i}(s)\right) M_{i}$ and $t-h_{i}(t)$ respectively for $t, r, s \in I$ in which $M_{i}$ are given by $(4.2)$.

Proof. Let $u(t) \in C(I, R)$ be a solution of (4.1). Then from (4.1), (4.3) and (4.4) we have

$$
|x(t)| \leq c+\int_{t_{0}}^{t} \sum_{i=1}^{n} k_{i}(t, s) \mid x\left(s-h_{i}(s) \mid d s\right.
$$

for $t \in I$. By making the change of variables on the right hand side of (4.6) and using (4.2) we have

$$
|x(t)| \leq c+\sum_{i=1}^{n} \int_{t_{0}-h_{i}\left(t_{0}\right)}^{t_{0}-h_{i}(t)} k_{i}\left(t, \sigma+h_{i}(s)\right) M_{i}|x(\sigma)| d \sigma
$$

for $t \in I$. Now a suitable application of the inequality given in Theorem 2.1, part $\left(\mathrm{a}_{1}\right)$ to (4.7) yields the required estimate in (4.5).

The next theorem deals with the uniquness of the solution of equation (4.1) by assuming that the function $F$ satisfies the general Lipschitz condition.

Theorem 4.2. Suppose that the function $F$ in (4.1) satisfies the condition

$$
\left|F\left(t, s, u_{1}, \ldots, u_{n}\right)-F\left(t, s, v_{1}, \ldots, v_{n}\right)\right| \leq \sum_{i=1}^{n} k_{i}(t, s)\left|u_{i}-v_{i}\right|
$$

where $k_{i}(t, s)$ are as in Theorem 2.1. Then (4.1) has atmost one solution on I.

Proof. Let $u(t)$ and $v(t)$ be two solution of (4.1) on $I$, then we have

$$
\begin{aligned}
u(t)-v(t)=\int_{t_{0}}^{t}\left\{F\left(t, s, u\left(s-h_{1}(s)\right), \ldots, u\left(s-h_{n}(s)\right)\right)\right. \\
\left.\quad-F\left(t, s, v\left(s-h_{1}(s)\right), \ldots, v\left(s-h_{n}(s)\right)\right)\right\} d s .
\end{aligned}
$$


Using (4.8) in (4.9), making the change of variables and using (4.2) we have

$$
\begin{aligned}
|u(t)-v(t)| & =\int_{t_{0}}^{t} \sum_{i=1}^{n} k_{i}(t, s)\left|u\left(s-h_{i}(s)\right)-v\left(s-h_{i}(s)\right)\right| d s \\
& \leq \sum_{i=1}^{n} \int_{t_{0}}^{t-h_{i}(t)} k_{i}\left(t, \sigma+h_{i}(s)\right) M_{i}|u(\sigma)-v(\sigma)| d \sigma
\end{aligned}
$$

for $t \in I$. A suitable application of the inequality given in Theorem 2.1, part ( $\left.\mathrm{a}_{1}\right)$ to (4.10) yields $|u(t)-v(t)| \leq 0$. Therefore $u(t)=v(t)$, i.e. there is at most one solution to (4.1) on $I$.

\section{References}

[1] D. Bainov and P. Simeonov, Integral Inequalities and Applications, Klumer Academic Publishers, Dordrecht, 1992.

[2] S. S. Dragomir, Some Gronwall Type Lemmas and Applications, RGMIA Research monographs, Victoria University of Technology, 2001.

[3] O. Lipovan, A retarded Gronwall-like inequality and applications, J. Math. Anal. Appl. 252(2000), 389-401.

[4] B. G. Pachpatte, Inequalities for Differential and Integral Equations, Academic Press, New York, 1998.

[5] B. G. Pachpatte, Explicit bounds on certain integral inequalities, J. Math. Anal. Appl. 267(2002), 48-61.

[6] B. G. Pachpatte, On some retarded integral inequalities and applications, J. Inequal. pure and Appl. Math. 3(2002), Art.18.

57, Shri Niketen Coloney, Aurangabad-431001, Maharashtra, India.

Email: bgpachpatte@hotmail.com 